\title{
Three-dimensional kidney's stones segmentation and chemical composition detection
}

\author{
Hiam Alquran', Mohammed Alslity ${ }^{2}$, Isam Abu Qasmieh ${ }^{3}$, Khaled Z. Alawneh, \\ Ali Mohammad Alqudah ${ }^{5}$, Ahmed Al-Rasheed ${ }^{6}$, Mohammed Al-Hawari ${ }^{7}$ \\ 1,2,3,5,6,7 Department of Biomedical Systems and Informatics Engineering, Yarmouk University, Irbid, Jordan \\ ${ }^{4}$ Faculty of Medicine, Jordan University of Science and Technology, Irbid, Jordan \\ ${ }^{4}$ King Abdullah University Hospital, Irbid, Jordan
}

\begin{tabular}{|c|c|}
\hline Article Info & ABSTRACT \\
\hline Article history: & Kidney stones are a common and extremely painful disease and can affect \\
\hline Received Sep 7, 2020 & $\begin{array}{l}\text { any part of the urinary tract. Ultrasound and computed tomography }(\mathrm{CT}) \text { are } \\
\text { the most frequent imaging modalities used for patients with acute flank pain. }\end{array}$ \\
\hline Revised Mar 24, 2021 & In this paper, we design an automated system for 3D kidney segmentation \\
\hline Accepted Apr 7, 2021 & $\begin{array}{l}\text { and stones detection in addition to their number and size evaluation. The } \\
\text { proposed system is built based on CT kidney image series of } 10 \text { subjects, }\end{array}$ \\
\hline Keywords: & $\begin{array}{l}\text { four healthy subjects (with no stones) and the rest have stones based on } \\
\text { medical doctor diagnosis, and its performance is tested based on } 32 \text { CT }\end{array}$ \\
\hline 3D reconstruction & kidney series images. The designed system shows its ability to extract kidney \\
\hline Convex hull & either in abdominal or pelvis non-contrast series CT images, and it \\
\hline $\mathrm{CT}$ & $\begin{array}{l}\text { distinguishes the stones from the surrounding tissues in the kidney image, } \\
\text { besides to its ability to analyze the stones and classify them in vivo for }\end{array}$ \\
\hline CT-windowing & further medical treatment. The result agreed with medical doctor's diagnosis. \\
\hline $\mathrm{HU}$ & The system can be improved by analyzing the stones in the laboratory and \\
\hline Kidney stones & $\begin{array}{l}\text { using a large CT dataset. The present method is not limited to extract stones } \\
\text { but, also a new approach is proposed to extract the 3D kidneys as well with } \\
\text { accuracy } 99 \% \text {. }\end{array}$ \\
\hline
\end{tabular}

This is an open access article under the CC BY-SA license.

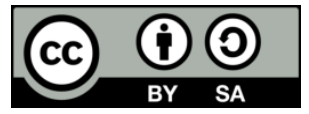

\section{Corresponding Author: \\ Hiam Alquran \\ Department of Biomedical Systems and Informatics Engineering \\ Yarmouk University \\ 566 Shafit Irshidat Street, Irbid 21163, Jordan \\ Email: heyam.q@yu.edu.jo}

\section{INTRODUCTION}

The renal stone disease is a common clinical problem, it represents about $10-14 \%$ of the population around the world. Renal stones can vary based on their chemical composition to around sixteen types. The major chemical composition is calcium oxalate which forms around $70 \%$, while calcium phosphate is around $20 \%$, and the rest are uric acid with approximately $8 \%$ and cystine $2 \%$ [1], [2]. Severe problems may maybe resulted from urinary stones if they untreated., such as; urosepsis and pyelonephritis. Therefore, a longstanding urinary obstruction may lead to a renal deficiency which ended with renal disease [3].

The characterizes of the urinary stones such as shape, chemical composition and size will help the clinician to specify the most suitable treatment. For instant, stones that are formed from uric acid don't always need surgery [4]. Kidney stones imaging is a crucial diagnostic tool and it is the initial step in determining which therapeutic options are the most suitable. Also, using the CT without adding contrast is the most accurate imaging modality for kidney stones due to its high sensitivity, specificity, and accurate stone sizing [5]. 
The advantage of utilizing ultrasonography in stone detection over CT, is its noninvasiveness because the absence of ionizing radiation, beside its cost effectiveness over CT, yet it has several limitations, such as lower sensitivity and specificity [6]. CT is based on the fundamental principle that the tissue density can be measured from the calculation of the attenuation coefficients, which are a tissue characteristic values [7]. Using this principle, CT allows constructing of the body tissue density, by reconstructing two-dimensional section image perpendicular to the axis of the acquisition system [8].

Hounsfield unit is the dimensionless and it is used in CT scanning to define the CT number in standard form. it is resulted from linear transformation of the attention coefficient of the imaging subject [9]. Image segmentation is one of the most critical issues in medical imaging technology. It helps the physicians in different ways, diagnosis, analysis and specifying the suitable treatment [10].

Many approaches for kidney segmentation have been developed over recent years. Cuingnet et al. [11] employed random regression and classification forests to detect and segment kidneys in CT images, automatically. On the other hand, Song et al. [12] extracted the kidneys as well, by combining SKFCM and improved GrowCut algorithms. He tested his approach on abdominal CT images. Tsagaan and Shimizu [13] utilized the kidney shape and its gray level appearance to segment it automatically.

Khalifa [14] employed Random Forest classifier for 3D CT kindeney segmenation automatically, with volumetric accuracy reached to $95 \%$. On the other hand, Cruz [15] utilized the benefits of deep learning techniques in kidney's segmentation with accuracy reached to 99.92\%. Mishr [16], utilized gray level cooccarnce matrix to extract features beside fuzzy c-mean clustering for kidney's stones segmentation, their performance is evaluated using back propagation neural network in terms of accuracy. The present study has built on previous research work in extracting kidneys and in detecting the existed stones.

In this paper, we propose a method that uses the CT principle for kidney segmentation from other slice image objects and to detect the stones automatically. The proposed stones detection system could quantify their sizes, number and locations in detail. The rest of this paper is organized as follows: Section two explains the research method, section three describes the results of the proposed technique and section four discusses the results' analysis. While section five we conclude the paper with future work.

\section{RESEARCH METHOD}

The proposed system for kidney image segmentation is summarized in the block diagram shown in Figure 1. The present system is implemented for the left and right kidney segmentation from the 2D abdominal CT series images.

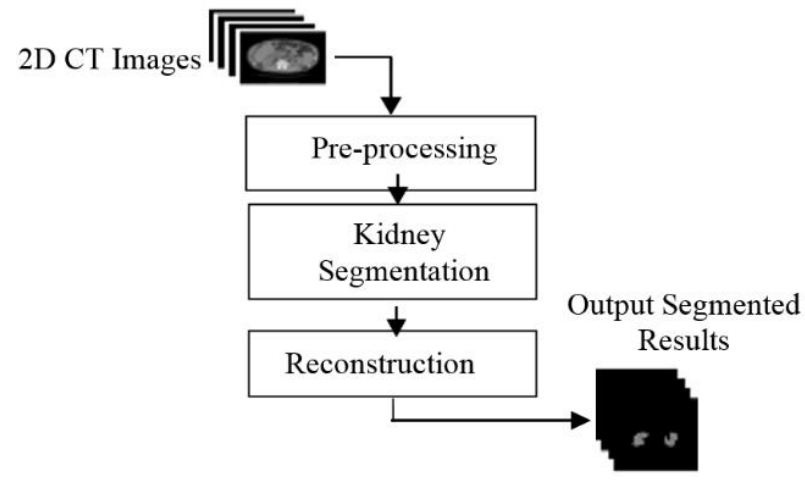

Figure 1. Flow diagram of the proposed method

\subsection{Data acquisition}

The protocol that has been followed in this paper is, the patient should be in supine position, his arms above the head, and his breath is hold during the acquisition. Figure 2 represents the position. In order to minimize the radiation, the patients were scanned with a standard low-dose renal stone CT protocol, using a tube potential of $120 \mathrm{kVp}$ and very low tube current 150-200 mAs, with slice thickness: $3 \mathrm{~mm}$ [17]. The protocol is applied to 42 subjects, the images of 10 subjects have been used to build the system and the rest for testing stage. The number of CT slices for each case is 140-150 slices. The 3D view of the abdominal slices for supine position is shown in Figure 2. We could not visualize 3D kidney segment and its stones using whole abdominal CT images. Therefore, image processing techniques have been applied to exclude kidney from the whole CT series images to facilitate the kidney diagnosis and the analysis of its components.

Three-dimensional kidney's stones segmentation and chemical composition detection (Hiam Alquran) 


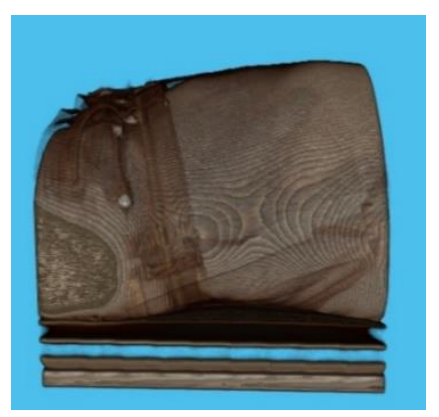

Figure 2. 3D view of abdominal slices

\subsection{Preprocessing}

The pre-processing technique is applied to the images to enhance their appearance and to make them suitable for further processes [18]. Figure 3 describes the pre-processing steps.

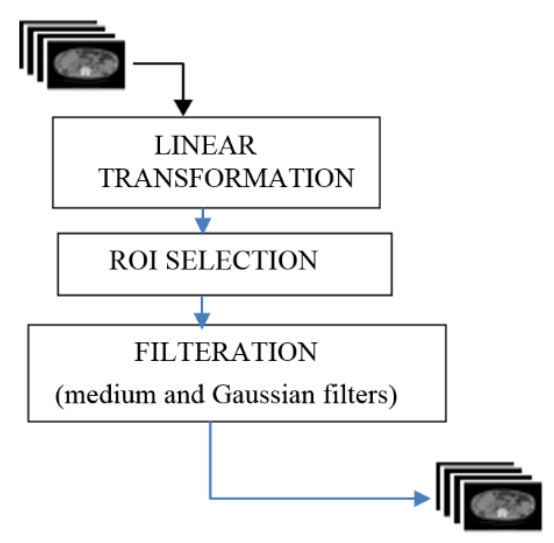

Figure 3. Pre-processing steps

\subsubsection{Linear transformation}

The module DICOMRescale allows the rescaling of DICOM CT data for defining intercept, slope, and data type. The intercept and slope define the transformation between intensity values/attenuation coefficients and Hounsfield Units (HU) used for displaying CT image data [19].

$$
H U=I V \times \text { slope }+ \text { intercept }
$$

This equation can also be used to scale any incoming CT series data into an unsigned 8-bit image for more processing and still have the correct correspondence of intensity values and HU [19].

\subsubsection{ROI selection}

Determination of the region of interest (ROI) is obtained by image binarization and then finding the largest rectangle inside the binary-sub matrix [20], followed by cropping the image by multiplying the white rectangle with the original series. We performed this operation to eliminate the surrounding tissues to the kidneys.

\subsubsection{Filtration}

A median filter is used with window size $3 \times 3$ to reduce the spatial noise, and it is followed by a Gaussian filter which is utilized here to reduce the high spatial frequency noise for further image enhancement and improving image quality.

\subsection{Segmentation}

The most straightforward segmentation method is thresholding, which is based on selecting a suitable intensity value threshold, all values higher than the selected one are classified as foreground and the 
rest are classified as background. In our case, the histogram would show three distributions, the intensity with intermediate image gray values needs to be separated from the darker background and the brighter intensities, The intermediate distribution can be separated utilizing the two thresholds T1 and T2, where T1<T2. The image with the threshold feature is then converted into a binary mask. Erosion is one of the two basic operators in the field of image morphology processing, the other being dilation. Thus, the areas of foreground pixels shrink in size, and holes within those areas become larger [21]. It is typically applied to the binary image.

\subsubsection{Extract objects}

Based on the area of the objects (which is the number of pixels that represented each object) we decide to preserve it in the output image or filtrate it.

\subsubsection{Convex hull}

The convex hull of a set of points is defined as the smallest convex polygon, that encloses all the points in the set.

\subsection{The 3D reconstruction}

Reconstruction of 3D volume image using 2D CT slices provides many advantages to medical doctors. The important step to achieve this goal is matching corresponding key points in the input CT slices in order to estimate the final 3D model. Object's detection and studying their features, such as geometrical properties and 3D features is not easy task in many vision systems [22]. Figure 4 shows the 3D reconstruction of kidney from different perspective.
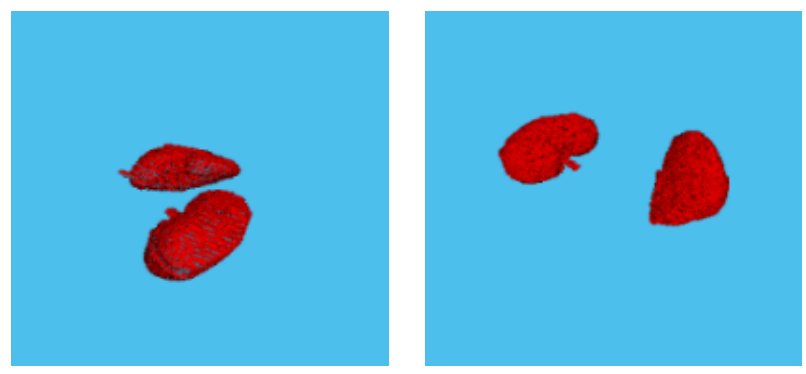

Figure 4. 3D view of both right and left kidneys

\subsection{Stone detection}

Once the 3D kidneys images have been segmented from the CT, the analysis can be performed. Intensity features has been used. It concerns the intensity of the voxels inside the 3D volume Gray level inhomogeneity distribution inside the objects maybe lead to use intensity based measurement. Figure 5(a) and (b) describe the appearance of the 3D stones in the kidney.

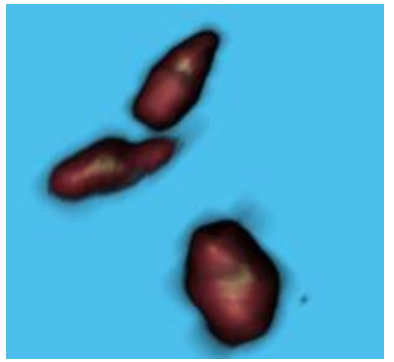

(a)

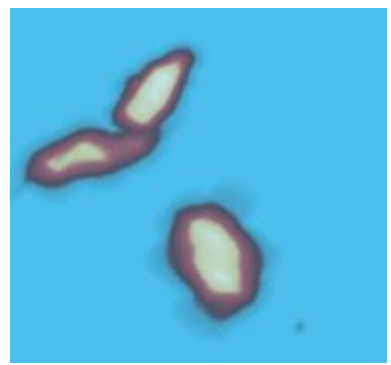

(b)

Figure 5. 3D reconstruction of kidney stones

The properties of the detected stones are evaluated based on pixel-based and statistical analysis, beside to the geometrical shape features for each detected stone: 


\subsubsection{Pixel-based measures}

Most often, the stones encountered in vivo are of mixed composition, the most common chemical composition that forms stones are calcium (90\%), uric acid (8\%) and cysteine (2\%) [23]. The researchers recently reported that calcium oxalate, uric and cystine stones could be marginally distinguished [24]. Intensity features concern the object measures reflect the intensity of the voxels inside the object. The stone composition can be determined using HU, CT Attenuation values. The stone composition can be determined using HU, CT Attenuation values [25].

In this study, we extracted the stones from 3D kidney volume by using the intensity-based measure. The stone composition can be determined using HU. Table 1 contains HU value for most common kidney stones [2], [3].

Table 1. Stone composition determined using HU

\begin{tabular}{cc}
\hline Stone Composition & Attenuation value at $120 \mathrm{kVp}$ \\
\hline Uric Acid & $200-450 \mathrm{HU}$ \\
Calcium Phosphate & $450-1000 \mathrm{HU}$ \\
Cystine & $1000-1600 \mathrm{HU}$ \\
Struvite & up-1500 HU \\
\hline
\end{tabular}

\subsubsection{Statistical analysis}

Most often, in vivo, the stones are made up of mixed composition. Therefore, we used statistical analysis to determine whether it is made of one type or more. To achieve this goal, we used the percentage HU to express the ratio of stone compositions; categorical variables of each type of stones were expressed as percentages. It gives the percentage value of the voxels in the image.

$$
\text { percentages } H U=\frac{\text { Number } H U \text { value of object }}{\text { Total number of voxels of object }}
$$

\subsubsection{Geometrical features of stones}

The geometrical features concern the shape of the object without considering its intensity values. Basic measures are length and location by finding connected components in the binary image. The geometrical analysis of the extracted stones is performed based on pixel spacing. Pixel spacing is determined physical distance between the centers of two-dimensional pixels, and it is expressed by two values. The first value is row spacing in $\mathrm{mm}$ and the second value is column spacing [26].

\section{RESULTS AND DISCUSSION}

The Graphical Interface system is designed, so, the series of CT images can be uploaded, then the designed system will next extract the image segments of the two kidneys and the stones inside. After that, the system provides to the user the number, composition, location, and dimension of the stones automatically. The system is trained on 10 cases, 6 of them have kidney stones either one type or multi-types o stones. Beside to 4 of them without kidney stones. After that, the system is tested on 32 cases of abdominal CT images, Table 2 describes each case, number of stones and their sizes in each side, beside to the ground truth for each one individually.

As demonstrated in Table 2, the proposed system has achieved its ability to illustrate the presence or absence of stones beside to their dimensions. The system results showed the agreement with physician's report, and it provides more details about the stones in each side comparing to that provided by the physician report. In many cases as explained in Table 2, the report registered the stones with "multi", while the system gives accurate number of stones. On the other hand, in the physician report the dimension of the stone is denoted by "NAN", whereas, the system specifies the stone's dimension in detail.

In many cases the system agreed with the doctor's report. The last three cases, the system detect the stones in the right side, and it agrees with doctor's result. Due to the placement of "Double-j" ureteral catheter, as reported by the clinicians in the left side of the kidney, the proposed system couldn't detect any stones or the kidney itself. Beside to location and dimension of the stone, the system can give preliminary result about the composition of the detected stones in vivo. This advantage is not reported by the clinicians unless the stones are being extracted and tested in-vitro (Clinical Tests).

On the other hand, 3D kidney's reconstruction accuracy is evaluated based on comparing between the automated kidneys segmented with that is resulted from manual segmentation. Pearson's coefficient is computed for all 3D segmented volumes, between manual and their corresponding automated. The average 
accuracy for all cases is $99 \%$. The following Table 3 compares the obtained results with that are mentioned in the literature review section.

Table 2. Real test dataset for different cases results

\begin{tabular}{|c|c|c|c|c|c|c|c|c|}
\hline \multirow{3}{*}{ ID } & \multicolumn{4}{|c|}{ Right kidney } & \multicolumn{4}{|c|}{ Left kidney } \\
\hline & \multicolumn{2}{|c|}{ \# of stone } & \multicolumn{2}{|c|}{ Largest measure $(\mathrm{cm})$} & \multicolumn{2}{|c|}{ \# of stone } & \multicolumn{2}{|c|}{ Largest measure $(\mathrm{cm})$} \\
\hline & Doctor & Present work & doctor & Present work & doctor & Present work & doctor & Present work \\
\hline 2342 & 2 & 1 & NAN & $0.2 * 0.1$ & No stone & No stone & No stone & No stone \\
\hline 10810 & Multi & 5 & 0.5 & $0.4 * 0.3$ & multi & 4 & 0.55 & $0.6 * 0.4$ \\
\hline 10865 & 4 & 2 & $0.4 * 0.2$ & $0.3 * 0.2$ & 4 & 3 & $1 * 0.8$ & $0.9 * 0.8$ \\
\hline 14471 & No stone & No stone & No stone & No stone & No stone & No stone & No stone & No stone \\
\hline 28296 & No stone & No stone & No stone & No stone & 1 & 1 & 0.26 & $0.2 * 0.1$ \\
\hline 28399 & 2 & 1 & 0.4 & $0.3 * 0.2$ & 1 & 1 & 0.2 & $0.2 * 0.1$ \\
\hline 32003 & No stone & No stone & No stone & No stone & No stone & No stone & No stone & No stone \\
\hline 33936 & No stone & No stone & No stone & No stone & 1 & 1 & $1.5^{*} 1$ & $1.5^{*} 1.1$ \\
\hline 38750 & No stone & No stone & No stone & No stone & No stone & No stone & No stone & No stone \\
\hline 40855 & No stone & No stone & No stone & No stone & No stone & No stone & No stone & No stone \\
\hline 40974 & No stone & No stone & No stone & No stone & No stone & No stone & No stone & No stone \\
\hline 41263 & No stone & No stone & No stone & No stone & few & NAN & 4 & $0.3 * 0.2$ \\
\hline 42364 & 1 & 1 & NAN & $0.1 * 0.1$ & No stone & No stone & No stone & No stone \\
\hline 43027 & 1 & 1 & NAN & $0.3^{*} 0.2$ & No stone & No stone & No stone & No stone \\
\hline 45649 & 1 & 1 & $1.7 * 1$ & $1.7 * 1$ & No stone & No stone & No stone & No stone \\
\hline 48142 & No stone & No stone & No stone & No stone & 1 & 1 & 1.6.*0.9 & $1.5^{*} 0.9$ \\
\hline 53466 & No stone & No stone & No stone & No stone & 1 & 1 & 0.5 & $0.4 * 0.2$ \\
\hline 55030 & 1 & 1 & NAN & $0.1 * 0.1$ & Few & 3 & 0.3 & $0.3 * 0.1$ \\
\hline 64797 & No stone & No stone & No stone & No stone & 1 & 1 & 0.2 & $0.2 * 0.1$ \\
\hline 67271 & No stone & No stone & No stone & No stone & No stone & No stone & No stone & No stone \\
\hline 67367 & No stone & No stone & No stone & No stone & 1 & No stone & $1.5 * .6$ & No stone \\
\hline 67379 & No stone & No stone & No stone & No stone & No stone & No stone & No stone & No stone \\
\hline 68630 & 1 & 1 & 0.3 & $0.3 * 0.2$ & No stone & No stone & No stone & No stone \\
\hline 68890 & 1 & 1 & $0.2 * 0.1$ & $0.2 * 0.1$ & 1 & 1 & $0.2 * 0.2$ & $0.2 * 0.2$ \\
\hline 69061 & No stone & No stone & No stone & No stone & 1 & 1 & 0.2 & $0.2 * 0.1$ \\
\hline 149937 & No stone & No stone & No stone & No stone & 1 & 1 & $0.2 * 0.2$ & $0.2 * 0.2$ \\
\hline 150246 & 4 & 4 & $2 * 1.5$ & $2.1 * 1.5$ & No stone & No stone & No stone & No stone \\
\hline 150638 & Multi & 4 & 0.5 & $0.5^{*} 0.2$ & Multi & 3 & 0.6 & $0.5^{*} 0.4$ \\
\hline 150534 & No stone & No stone & No stone & No stone & No stone & No stone & No stone & No stone \\
\hline \multicolumn{9}{|c|}{ Abnormal cases } \\
\hline 26168 & 2 & 2 & $0.6 * 0.7$ & $0.7 * 0.5$ & Double-j & Double-j & Double-j & Double-j \\
\hline 41171 & Few & 3 & 0.5 & $0.4 . * 0.2$ & Double-j & Double-j & Double-j & Double-j \\
\hline $6853-2$ & No stone & No stone & No stone & No stone & Double-j & Double-j & Double-j & Double-j \\
\hline
\end{tabular}

Table 3. Comparison of the obtained results with literature review

\begin{tabular}{cc}
\hline Methods & Accuracy $\%$ \\
\hline H. Song & $99.66 \%$ \\
Tsagaan and Shimizu & $87 \%$ \\
Fahmi Khalifa & $95 \%$ \\
Luana Batistada Cruz & $99.92 \%$ \\
Riya Mishr & $98.8 \%$ \\
Proposed Method & $\mathbf{9 9 \%}$ \\
\hline
\end{tabular}

All literature review results were obtained for kidney segmentation such as [12]-[15], but Riya Mishr detected the kidney's stones. The presented work focused on both, volumetric kidney's segmentation beside the stone's detection and analysis, in addition to expectation to their composition. All previous studies did not mention about the time required for the obtaining the goal, either kidney segmentation or stones detection. The proposed method computed the time required from starting the pre-processing the CT-series to achieving the results (3D kidney segmentation, stones location, sizes, and their composition) is less than 1 minute. The proposed method accurate, fast, and reliable because it works well on test data.

\section{DISCUSSION}

The main objective of this paper is the extraction of the 3D kidney segment from the CT images using various image processing techniques. In the proposed approach, we utilized abdominal CT images. Such these images provide a good abdominal view, but it fails to obtain an accurate view of the kidney. Therefore, various image processing techniques are employed to find out the exact position of the kidney and 
extract it. Image processing technique like image segmentation, and morphological operations. are applied. This helps to obtain a better insight into the kidney segment in CT images, which leads to facilitate detection of stones and to study their properties. Ureteric stones were excluded from the current study because their chemical composition has no role in kidney stones treatment, and the concern is mainly about their sizes rather than their chemical composition.

Clinical urinary tract stones management depends on several factors, such as stones location, their sizes, and their composition. Some types are difficult to eliminate due to their large size such as monohydrate calcium oxalate stones. In this paper we focused on using size and chemical composition in the treatment strategy. Renal stones less than $6 \mathrm{~mm}$ is treated by medical treatment, uric acid stones greater than $5 \mathrm{~mm}$ in diameter is treated by medical treatment, non-uric stones more than $20 \mathrm{~mm}$ is treated by percutaneous nephrolithotomy, and known uric stones 6 to $<16 \mathrm{~mm}$ in diameter is treated by extracorporeal shock wave lithotripsy (ESWL) [24]. Other treatment strategies are based on shape. Homogenous stone is more resistant to extracorporeal shock wave lithotripsy (ESWL) than heterogenous stone, because the last one is more fragile than that.

\section{CONCLUSION AND FUTURE WORK}

Provide a statement that what is expected, as stated in the "introduction" chapter can ultimately result in "results and discussion" chapter, so there is compatibility. Moreover, it can also be added the prospect of the development of research results and application prospects of further studies into the next (based on result and discussion). The kidney stone disease is a common clinical problem, Different chemical compounds forming kidney stones and the most common chemical composition that forms stones are calcium oxalate $(70 \%)$, calcium phosphate $(20 \%)$, uric acid $(8 \%)$ and cysteine $(2 \%)$. Deciding the appropriate kidney's stone therapeutic procedure needs kidney stone imaging as a first step. CT imaging modality is the most common standard for that purposes.

A proposed algorithm might assist in deciding on the best approach for imaging patients suspected of having a stone. In the current study, the assessment of renal stones attenuation profiles depends on their corresponding HU units. It provides the needed anatomical information like number, location, and maximal diameter. Also, it characterizes the stone chemical composition which consequently determines the optimal fragmentation method. Determination the chemical type accurately facilitate the selection of treatment option, For instance, some stone may not need surgery such as those composed of uric acid.

Using the CT number abdominal window, stents and stones may have the same CT appearance. However, the bone window allows a visual distinction between a stent/tube and a stone. In the present study, we excluded Ureteric stones analysis due to their chemical composition. The system could not recognize the kidney in cases that have catheter. Therefore, in future work another set of features will be used to segment the kidney image region, which does not depend on HU number only, but rather on another features such as those relevant to shape or texture, beside to the utilization of machine learning benefits to achieve the desired goal. This leads to generalize the usage of the proposed system in hospitals as a preliminary diagnosis tool for kidney stones detection and type recognition

\section{REFERENCES}

[1] Goldman L., M. D., Andrew I. Schafer, M. D., "Goldman-Cecil Medicine," Elsevier eBook on VitalSource, 2Volume Set, 25th Edition, 2016.

[2] Saita A., Bonaccorsi A., Motta M., "Stone composition: where do we stand?," Urol Internationalis, vol. 79, no. 1, pp. 16-19, 2016, doi: 10.1159/000104436.

[3] Cuhan G. C., "Epidemiology of stone diseases," Urologic Clinics of North America, vol. 34, no. 3, pp. 287-293, 2007, doi: 10.1016/j.ucl.2007.04.003.

[4] J. C. William, K. C. Saw, R. F. Paterson, E. K. Hatt, J. A McAteer, J. E. Lingeman, "Variability of renal stones fragility in shock wave lithotripsy," Urology, vol. 61, no. 6, pp. 1092-1096, 2003, doi: 10.1016/s00904295(03)00349-2.

[5] C. W. Fwu, P. W. Eggers, P. L. Kimmel, J. W. Kusek, and Z. Kirkali, "Emergency department visits, use of imaging, and drugs for urolithiasis have increased in the United States," Kidney International, vol. 83, no. 3, pp. 479-486, 2013, doi: 10.1038/ki.2012.419.

[6] R. Smith-Bindman, C. Aubin, J. Bailitz, R. N. Bengiamin, Jr. C. A. Camargo, J. Corbo et al., "Ultrasonography versus computed tomography for suspected nephrolithiasis," The New England Journal of Medicine, vol. 371, pp. 1100-1110. 2014, doi: 10.1056/NEJMoa1404446

[7] W. Huda, Richard M. Slone, "Review of Radiologic Physics," Lippincott Williams and Wilkins; 2015

[8] Lazic J, Sobic V, Cikaric S et al., "Radiologija (Radiology-Unviersity Manual)," Medicinska Knjiga, Belgrade 1997. 
[9] G. N. Hounsfield "Computed medical imaging," Science, vol. 210, no. 4465, pp. 22-28, 1980, doi: 10.1126/science.6997993.

[10] Pham D. L., Xu C., Prince J. L., “Current methods in medical image segmentation,” Annual Review of Biomedical Engineering, vol. 2, pp. 315-338, 2000, doi: 10.1146/annurev.bioeng.2.1.315.

[11] R. Cuingnet, R. Prevost and D. Lesage, L. D. Cohen, "Automatic Detection and Segmentation of Kidneys in 3D CT Images Using Random Forests, UMR 7534 CNRS," International Conference on Medical Image Computing and Computer-Assisted Intervention, vol. 7512, 2012, pp. 66-74.

[12] H. Song W. Kang and Q. Zhang, "Kidney segmentation in CT sequences using SKFCM and improved GrowCut algorithm," BMC Systems Biology, vol. 9, no. S5, 2015, doi: 10.1186/1752-0509-9-S5-S5.

[13] Tsagaan B., Shimizu A., Kobatake H., Miyakawa K., Hanzawa Y., "Segmentation of kidney by using a deformable model," Proceedings 2001 International Conference on Image Processing (Cat. No.01CH37205), Thessaloniki, Greece, vol. 3, 2001, pp. 1059-1062, doi: 10.1109/ICIP.2001.958309

[14] Khalifa, Fahmi, Ahmed Soliman, Adel Elmaghraby, Georgy Gimel'farb, Ayman El-Baz, "3D kidney segmentation from abdominal images using spatial-appearance models," Computational and mathematical methods in medicine, vol. 2017, 2017, doi: 10.1155/2017/9818506.

[15] Da Cruz, Luana Batista, José Denes Lima Araújo, Jonnison Lima Ferreira, João Otávio Bandeira Diniz, Aristófanes Corrêa Silva, João Dallyson Sousade Almeida et al., "Computers in Biology and Medicine, vol. 123, 2020, Art. No. 103906, doi: 10.1016/j.compbiomed.2020.103906

[16] R. Mishr, A. Bhattacharjee, M. Gayathri, C. Malathy, "Kidney Stone Detection with CT Images Using Neural Network," International Journal of Psychosocial Rehabilitation, vol. 24, no. 8, pp. 2490-2497, 2020, doi: 10.37200/IJPR/V24I8/PR280269.

[17] A. Heidenreich, F. Desgrandschamps, and F. Terrier, "Modern approach of diagnosis and management of acute flank pain: review of all imaging modalities," Europenan Urology, vol. 41, no. 4, pp. 351-362, 2002, doi: 10.1016/s0302-2838(02)00064-7.

[18] B. Chitradevi, P. Srimathi, "An Overview on Image Processing Techniques," 2012. [Online]. Available: https://www.rroij.com.

[19] Corl F. M., Garland M. R., Lawler L. P., Fishman E. K., "A five-step approach to digital image manipulation for the radiologist," Radiographics, vol. 22, no. 4, pp. 981-992, 2002 doi: 10.1148/radiographics.22.4.g02j133981.

[20] Abu-Qasmieh, Isam, and Hiam Al-quran, "Unrestricted LR detection for biomedical applications using coarse-tofine hierarchical approach," IET Image Processing, vol. 12, no. 9, pp. 1639-1645, 2018, doi: 10.1049/ietipr.2017.0509.

[21] Geoff Dougherty, "Digital Image Processing for Medical Applications," California State University, Channel Islands, 2009.

[22] Pascal Sommer, "A gentle introduction to the convex hull problem," 2016, [Online]. Available: https://medium.com/@ pascal.sommer.ch/a-gentle-introduction-to-the-convex-hull-problem-62dfcabee90c.

[23] Howard J. D., Elster A. D., May J. S. "Temporal bone: three-dimensional CT. I. Normal anatomy, techniques, and limitations,” Radiology, vol. 177, no. 2, pp. 421-425, 1990, doi: 10.1148/radiology.177.2.2217779.

[24] "Center for Research in Hepatic and Renal Diseases," Federal University of Ceará-UFC, and Division of Nephrology2010.

[25] A. N. Primak, J. G. Fletcher, T. J. Vrtiska, O. P. Dzyubak, J. C. Lieske, M. E. Jackson et al., "Noninvasion differentiation of uric acid versus non uric acid kidney stones using dual energy C. T.," Academic Radiology, vol. 14, no. 12, pp. 1441-1447, 2007 doi: 10.1016/j.acra.2007.09.016.

[26] Department of Radiology, "Hôpital Paul-Brousse, AP-HP," Université Paris 11, 12-14 av P.V. Couturier, 94804 Villejuif Cedex, France. 